\title{
Macroion solutions in the cell model studied by field theory and Monte Carlo simulations
}

\author{
Leo Lue ${ }^{1, \text { a) }}$ and Per Linse ${ }^{2}$ \\ ${ }^{1)}$ Department of Chemical and Process Engineering, University of Strathclyde \\ James Weir Building, 75 Montrose Street, Glasgow G1 1XJ, \\ United Kingdom \\ 2) Physical Chemistry, Department of Chemistry \\ Lund University, P.O. Box 124, S-221 00 Lund, Sweden
}

(Dated: 29 October 2014)

Aqueous solutions of charged spherical macroions with variable dielectric permittivity and their associated counterions are examined within the cell model using a field theory and Monte Carlo simulations. The field theory is based on separation of fields into short- and long-wavelength terms, which are subjected to different statistical-mechanical approximations. The simulations were performed by using a new, accurate, and fast algorithm for numerical evaluation of the electrostatic polarization interaction. The field theory provides counterion distributions outside a macroion in good agreement with the simulation results over the full range from weak to strong electrostatic coupling. A low-dielectric macroion leads to a displacement of the counterions away from the macroion.

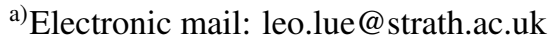




\section{INTRODUCTION}

Solutions of charged colloidal particles occur in nearly all biological systems and in many industrially important formulations. The nature of the electrical double layer composed of colloidal surface charges and its neutralizing mobile counterions has a large influence on structural and thermodynamic properties of these systems ${ }^{1}$.

The Poisson-Boltzmann (PB) theory has been successful in describing many properties of the electric double layer and is responsible for shaping much of our current understanding of the behavior of solutions containing charged colloids. This theory works best when the electrostatic interactions in the system are relatively weak, i.e., low colloidal surface charge density, monovalent counterions, high dielectric constant, and/or high temperature. The breakdown of the PB theory at strong electrostatic interactions is primarily due to its neglect of correlations between the counterions, which becomes increasingly important at such conditions.

Many theories have been developed to account for correlation effects, such as modified PB approaches $^{2-4}$, liquid state theories such as the hypernetted-chain and mean-spherical approximations ${ }^{5-7}$, loop expansions ${ }^{8,9}$, variational approximations ${ }^{10}$, to field theoretic formulations, and densityfunctional theories ${ }^{4,11,12}$. These different approaches improve upon the PB theory to varying degrees; however, they all tend to breakdown when the electrostatic interactions become even stronger. When the electrostatic interactions are extremely strong, a strong-coupling expansion method has been developed ${ }^{13,14}$ that accurately describes the electrostatic double layer.

Even though the PB theory is known to fail for highly electrostatically coupled systems, it has been found that it can still be used to quantitatively fit properties of these systems provided a renormalized charge is used (see Ref. 15 and references given therein). Such a use is sometimes physically justified by the strong attraction of counterions to the surface, which often are considered to be bound to the surface. Several theoretical approaches have been developed to relate the renormalized charge to the underlying stociometric charge of the colloids. The renormalized jellium model ${ }^{16-19}$, is such a relatively recent theoretical approach.

In the investigations referred to above, the dielectric constant of the colloids is the same as the surrounding solvent. However, in most aqueous colloidal solutions, the colloids have a dielectric constant lower than that of the surrounding solvent. This gives rise to an additional repelling force acting on the counterions arising from the surface charges induced at the dielectric discontinuity. Often this repulsion is viewed as arising from image charges (or image charge densities) located 
on the other side of the dielectric discontinuity as viewed from the counterions ${ }^{20}$.

Simulation of systems with dielectric discontinuities are computationally intensive, due to the many-body nature of the charge polarization at these discontinuities. Much of the early simulation work for systems with dielectric heterogeneities was limited to planar geometries where the imagecharge description becomes simple. However, in spherical geometry the image-charge casting leads to a varying linear image charge density, and the calculations become computationally much more involved. Consequently, there have been relatively few such studies ${ }^{21,22}$. In a very recent work, dos Santos et al. ${ }^{23}$ proposed a much faster algorithm that is accurate for low-dielectric spheres in a high-dielectric medium. Also recently, extenstions to other more complex geometries, such as pairs of interacting dielectric spheres ${ }^{24}$ and parallel cylinders ${ }^{25}$ have been made.

Different theoretical approaches have been developed to analyze systems with dielectric heterogeneities. First, due to the neglect of ion-ion correlations, in spherical geometry there are no additional image-charge forces in the PB theory. For the planar geometry, early approaches ${ }^{26,27}$ added the image-charge interaction directly to the PB theory as an additional external potential acting on the ions and this approach has been extended to weakly curved interfaces ${ }^{28}$. Later, these imagecharge interaction were included more rigorously in integral equation approaches, such as the hypernetted chain approximation ${ }^{29-32}$. Field-theoretic methods based on the loop expansion ${ }^{33-35}$ and variational approximation ${ }^{36}$ have also been applied to planar geometry. A variational fieldtheoretical approach has been used to study image-charge effects in spherical ${ }^{10}$ and cylindrical ${ }^{37}$ geometries and a loop expansion approach has been used to examine two interacting dielectric spheres $^{38}$. The approaches mentioned so far are only accurate for weak electrostatic coupling. A strong-coupling theory have been developed for strong electrostatic coupling. It has been expanded to include systems with dielectric heterogeneities, and this has been applied to systems in planar geometries ${ }^{39}$, for systems of parallel cylinders ${ }^{25,40}$, and spheres ${ }^{41}$.

In this work, we examine the properties of salt-free solutions of spherical macroions, including the effects of a low dielectric interior, within the context of the cell model. Recently, a simple approximate field theory has been developed ${ }^{42,43}$ that works well from the weak- through to the strong-coupling regimes, even for systems with dielectric heterogeneities. It relies on dividing the electrostatic interactions in the system into short and long-wavelength contributions and treating each within separate approximation schemes. While this theory can be used in arbitrary geometries, it has so far only been applied to planar systems, where it works fairly well. Here, we asses the predictions of this field-theory approach ${ }^{42,43}$ for the spherical cell model by comparing to es- 
sentially exact Monte Caro simulation data. The latter were obtained by using a new and more quickly converging expansion for the electrostatic energy of a system with a spherical dielectric interface. Our algorithm shares many features with the one by dos Santos et al. ${ }^{23}$, but our derivation is mathematically simpler and is formally exact for all values of the dielectric constant.

The cell model is described in the next section, and in Section III details of the systems that we study are given. Then in Section IV, we give a brief overview of the splitting theory and its application to the spherical cell model. Details of the simulation methods are provided in Section V. Section VI provides our numerical findings. The main conclusions of our work are summarized in Section VII.

\section{BACKGROUND}

\section{A. Electrostatic interaction energy}

Consider a system composed of a fixed charge density distribution and mobile point charges. The total charge density $\mathcal{Q}$ at $\mathbf{r}$ is given by

$$
\mathcal{Q}(\mathbf{r})=\Sigma(\mathbf{r})+\sum_{k} q_{k} \delta^{d}\left(\mathbf{r}-\mathbf{r}_{k}\right)
$$

where $\Sigma(\mathbf{r})$ denotes the fixed charge density distribution and where $q_{k}$ is the charge and $\mathbf{r}_{k}$ the position of a mobile point charge $k$, with $\delta^{d}$ being the $d$-dimensional Dirac delta function, here $d=3$. If the system is embedded in a spatially varying dielectric medium described by $\varepsilon(\mathbf{r})$, then the electrostatic interaction energy $U$ of the system is given by

$$
U=\frac{1}{2} \int d \mathbf{r} d \mathbf{r}^{\prime} \mathcal{Q}(\mathbf{r}) G_{0}\left(\mathbf{r}, \mathbf{r}^{\prime}\right) \mathcal{Q}\left(\mathbf{r}^{\prime}\right)-\frac{1}{2} \sum_{k} q_{k}^{2} G_{\text {free }}\left(\mathbf{r}_{k}, \mathbf{r}_{k}\right)
$$

where $G_{0}$ is the Green's function of Poisson's equation

$$
-\frac{1}{4 \pi} \nabla \cdot \varepsilon(\mathbf{r}) \nabla G_{0}\left(\mathbf{r}, \mathbf{r}^{\prime}\right)=\delta\left(\mathbf{r}-\mathbf{r}^{\prime}\right)
$$


and $G_{\text {free }}$ is the Green's function for a system in a medium with a uniform dielectric constant $\varepsilon$, which is equal to

$$
G_{\text {free }}\left(\mathbf{r}, \mathbf{r}^{\prime}\right)=\frac{1}{\varepsilon\left|\mathbf{r}-\mathbf{r}^{\prime}\right|}
$$

The Green's function of the system $G_{0}$ can be considered as a sum of (i) a contribution $G_{\text {free }}$ from a uniform system and (ii) a contribution $\Delta G_{0}$, which is the change due to heterogeneities of the dielectric medium according to

$$
G_{0}\left(\mathbf{r}, \mathbf{r}^{\prime}\right)=G_{\text {free }}\left(\mathbf{r}, \mathbf{r}^{\prime}\right)+\Delta G_{0}\left(\mathbf{r}, \mathbf{r}^{\prime}\right)
$$

The electrostatic interaction energy $U$ of the system can be decomopsed according to

$$
U=U_{\Sigma \Sigma}+U_{q \Sigma}+U_{q q}+U_{\mathrm{pol}}
$$

where $U_{\Sigma \Sigma}$ is the interaction energy of the fixed charge distribution, $U_{\Sigma q}$ the Coulomb interaction between the fixed charge distribution and mobile point charges, $U_{q q}$ the "direct" Coulomb interaction between the mobile point charges, and $U_{\text {pol }}$ the polarization contribution to the interaction between the mobile point charges. These different terms are related to the various Green's functions as

$$
\begin{aligned}
U_{\Sigma \Sigma} & =\frac{1}{2} \int d \mathbf{r} \int d \mathbf{r}^{\prime} \Sigma(\mathbf{r}) G_{0}\left(\mathbf{r}, \mathbf{r}^{\prime}\right) \Sigma\left(\mathbf{r}^{\prime}\right) \\
U_{q \Sigma} & =\sum_{k} u_{q \Sigma}\left(\mathbf{r}_{k}\right)=\sum_{k} q_{k} \int d \mathbf{r} G_{0}\left(\mathbf{r}_{k}, \mathbf{r}\right) \Sigma(\mathbf{r}) \\
U_{q q} & =\sum_{j<k} u_{q q}\left(\mathbf{r}_{j}, \mathbf{r}_{k}\right)=\sum_{j<k} q_{j} q_{k} G_{\text {free }}\left(\mathbf{r}_{j}, \mathbf{r}_{k}\right) \\
U_{\mathrm{pol}} & =\frac{1}{2} \sum_{j, k} u_{\mathrm{pol}}\left(\mathbf{r}_{j}, \mathbf{r}_{k}\right)=\frac{1}{2} \sum_{j, k} q_{j} q_{k} \Delta G_{0}\left(\mathbf{r}_{j}, \mathbf{r}_{k}\right)
\end{aligned}
$$

which also defines the quantities $u_{q \Sigma}\left(\mathbf{r}_{k}\right), u_{q q}\left(\mathbf{r}_{j}, \mathbf{r}_{k}\right)$, and $u_{\text {pol }}\left(\mathbf{r}_{j}, \mathbf{r}_{k}\right)$.

\section{B. The primitive model and the spherical cell model}

The primitive model of asymmetric electrolytes constitutes a firm basis for a statisticalmechanical description of solutions of charged macromolecules. Within this model, ionic species 
are represented by charged hard spheres differing in charge and size, whereas the solvent enters the model only through its relative dielectric constant. In this study, solutions contain only charged spherical macroions and their counterions, forming an electrically neutral system, are considered; no simple salt is added. This implies that we can make the further simplification that counterions are point-like, i.e., their hard-sphere radii are zero.

In the spherical cell model, the solution of the asymmetric electrolyte is divided into subvolumes, each containing one macroion and a corresponding amount of counterions and solvent. This model is most frequently used to examine the distribution of ions near a macroion and to obtain approximate thermodynamic results. The symmetry of the cell should follow closely the symmetry of the macroion. The obvious choice is a concentric spherical cell placed around the macroion. The influence of the remaining solution is taken into account in a mean-field manner. The cell model is most useful when the macroions repel each other and becomes less appropriate when they possess attractive interactions.

Usually, the dielectric constant of the macroion is taken to be equal to that of the solvent. However, here we will consider the case where the interior of the macroion possesses a dielectric constant lower than that of the solvent, which is a more realistic description for colloids in aqueous solutions.

Given the primitive and spherical cell models, the interaction energy $U$ of the system can be expressed as

$$
U=\sum_{k} u_{q \Sigma}\left(\mathbf{r}_{k}\right)+\sum_{j<k} u_{q q}\left(\mathbf{r}_{j}, \mathbf{r}_{k}\right)+\sum_{k} u_{\mathrm{ext}}\left(\mathbf{r}_{k}\right)+\frac{1}{2} \sum_{j, k} u_{\mathrm{pol}}\left(\mathbf{r}_{j}, \mathbf{r}_{k}\right)
$$

The Coulomb and excluded-volume interaction between the macroion and counterion $k, u_{q \Sigma}\left(\mathbf{r}_{k}\right)$, is given by

$$
u_{q \Sigma}\left(\mathbf{r}_{k}\right)=\left\{\begin{array}{c}
\infty, \text { for } r_{k}<R_{M} \\
\frac{Q q_{k}}{\varepsilon r_{k}}, \text { for } r_{k} \geq R_{M}
\end{array}\right.
$$

with $Q$ denoting the macroion charge, $q_{k}$ the ionic charge, $R_{M}$ the macroion radius, $\varepsilon$ the relative dielectric constant of the solvent, and $r_{k}$ the radial position of the counterion. The Coulomb interaction between counterions $j$ and $k, u_{q q}\left(\mathbf{r}_{j}, \mathbf{r}_{k}\right)$, is given by

$$
u_{q q}\left(\mathbf{r}_{j}, \mathbf{r}_{k}\right)=\frac{q_{j} q_{k}}{\varepsilon\left|\mathbf{r}_{j}-\mathbf{r}_{k}\right|}
$$


The confining external potential energy $u_{\text {ext }}$ acting on counterion $k$ becomes

$$
u_{\text {ext }}\left(\mathbf{r}_{k}\right)=\left\{\begin{array}{c}
0, \text { for } r_{k} \leq R_{\text {cell }} \\
\infty, \text { for } r_{k}>R_{\text {cell }}
\end{array}\right.
$$

where $R_{\text {cell }}$ denotes the radius of the spherical cell.

In the case that the macroion has the same dielectric constant as the surrounding solution (homogeneous dielectric solution), the polarization energy $u_{\text {pol }}$ is zero. However, if the macroion has a dielectric constant $\varepsilon^{\prime} \neq \varepsilon$ (heterogeneous dielectric solution), a polarization surface charge density will appear at the dielectric discontinuity and $u_{\text {pol }}$ becomes nonzero. For simplicity, let the dielectric discontinuity be spherical with radius $R_{d}$. Then, the potential energy involving the polarization surface charge density and the counterions can be cast as an ionic self-term $u_{\text {pol }}\left(\mathbf{r}_{k}, \mathbf{r}_{k}\right)$ and an ion-ion term $u_{\text {pol }}\left(\mathbf{r}_{j}, \mathbf{r}_{k}\right)$ with

$$
u_{\mathrm{pol}}\left(\mathbf{r}_{j}, \mathbf{r}_{k}\right)=\frac{q_{j} q_{k}}{\varepsilon} \sum_{l=1}^{\infty} \sum_{m=-l}^{l} \frac{4 \pi}{2 l+1}\left[\frac{\varepsilon-\varepsilon^{\prime}}{\varepsilon(1+1 / l)+\varepsilon^{\prime}}\right]\left(\frac{R_{d}^{2 l+1}}{r_{j}^{l+1} r_{k}^{l+1}}\right) Y_{l m}\left(\theta_{j}, \phi_{j}\right) Y_{l m}^{*}\left(\theta_{k}, \phi_{k}\right)
$$

applicable to both $j=k$ and $j \neq k$, where $\mathbf{r}=(r, \theta, \phi), Y_{l m}$ denotes the spherical harmonics, and ${ }^{*}$ the complex conjugate. In the following, the dielectric discontinuity will be placed at the hard-sphere surface of the macroion, i.e., $R_{d}=R_{M}$. This maximizes the effect of the polarization surface charge density. The interaction energy associated with the surface polarization can be re-expressed in several ways with different convergence properties and computational efficiency.

\section{SYSTEMS}

In this work, we consider four aqueous solutions containing macroions and their counterions, which were studied in Ref. 44. As our primitive model systems are composed only of macroions and point counterions, the system is fully specified by three reduced parameters. Here, we choose (i) the charge ratio $Q / q$, (ii) the electrostatic coupling parameter $\Xi$ defined by

$$
\Xi=\frac{|Q| l_{B}^{2}}{2 R_{M}^{2}}
$$


which is one parameter (of many choices) that characterizes the strength of the electrostatic coupling in the system, and (iii) the macroion volume fraction $\phi_{M}$ given by

$$
\phi_{M}=\frac{4 \pi R_{M}^{3}}{3} \rho_{M}
$$

where $\rho_{M}$ is the macroion number density. In Eq. (16), $l_{B}=\left(q / e_{0}\right)^{2} \lambda_{B}$ is a modified Bjerrum length, where $\lambda_{B}$ is the Bjerrum length

$$
\lambda_{B}=\frac{e_{0}^{2}}{\varepsilon k_{B} T}
$$

with $e_{0}$ the fundamental unit of charge, $k_{B}$ being the Boltzmann constant, and $T$ the absolute temperature. Physically, the modified Bjerrum length is the distance between two counterions at which their electrostatic interaction energy becomes equal to the thermal energy $k_{B} T$.

Table I provides the values of $Q / q, \Xi$, and $\phi_{M}$ of the four systems. The electrostatic coupling parameter $\Xi$ ranges from weak $(\Xi \ll 1)$ to strong $(\Xi \gg 1)$ coupling in steps of approximately one order of magnitude from System IV to Systems I and II to System III. The macroion volume fraction $\phi_{M}=0.01$ is used throughout, which implies a cell to macroion radius ratio $R_{\text {cell }} / R_{M}=$ 4.6416. Table I also provides typical values of $Q, q$, and $R_{M}$ for colloids in aqueous solution at ambient temperature consistent with the specifications of Systems I-IV. Thus, Systems I, II, and III could represent solutions of charged surfactant micelles with (i) radius $R_{M}=20 \AA$, where the micelle is weakly charged in System I and highly charged in Systems II and III and (ii) monovalent counterions in Systems I and II and divalent ones in System III. Finally, System IV could represent a solution of silica particles with radius $R_{M}=160 \AA$ with monovalent counterions. Note, the colloid charge in System IV is the same as in Systems II and III.

\section{THE SPLITTING THEORY}

\section{A. General}

This section provides a brief outline of an approximate field theory, which we refer to as the splitting theory, applied to a system composed of a fixed charge distribution $\Sigma$ and mobile point charges of magnitude $q$. For further details, see Ref. 43. The basic idea behind the theory is to convert the partition function of the system to a functional integral over two interaction fields: 
TABLE I. Specification of the four systems considered in terms of charge ratio $Q / q$, electrostatic coupling parameter $\Xi$, and macroion volume fraction $\phi_{M}$ as well as corresponding macroion charge $Q$, counterion charge $q$, and macroion radius $R_{M}$ for typical aqueous colloidal solutions at ambient temperature. ${ }^{a}$

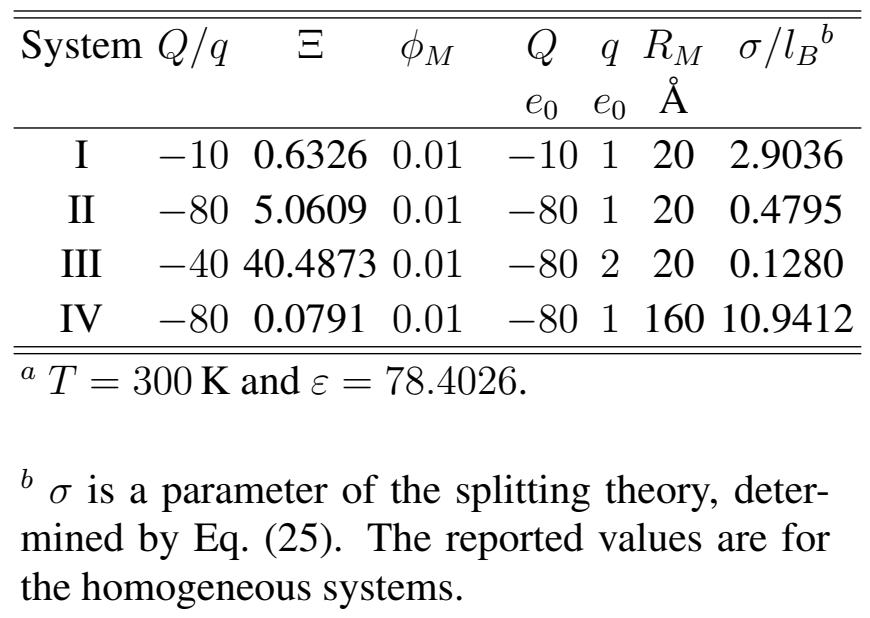

one varying over small wavelengths and the other varying over large wavelengths. The functional integration for each of these fields is evaluated using different approaches, viz. a virial expansion for the short-wavelength field and a mean-field approximation for the long-wavelength field. Thus, the variations in the electric potential due to the varying counterion configurations are at shortwavelengths taken into account but on long-wavelengths a mean-field description prevails.

The separation between short and long wavelength is achieved by dividing the Green's function $G_{0}$ of Poisson's equation according to

$$
G_{0}=G_{s}+G_{l}
$$

where $G_{l}=\mathcal{P} G_{0}$ and $G_{s}=(1-\mathcal{P}) G_{0}$ with $\mathcal{P}$ being an operator which splits the long-wavelength variations of the interaction field from the short-wavelength variations. The choice of the operator is somewhat arbitrary, and, in this work, we select $\mathcal{P}=\left[1-\sigma^{2} \nabla^{2}+\sigma^{4} \nabla^{4}\right]^{-1}$. The interaction energy $U$ can be rewritten in terms of these two Green's functions as

$$
\begin{aligned}
U=\frac{1}{2} \int d \mathbf{r} d \mathbf{r}^{\prime} \mathcal{Q}(\mathbf{r}) G_{l}\left(\mathbf{r}, \mathbf{r}^{\prime}\right) \mathcal{Q}\left(\mathbf{r}^{\prime}\right)+\frac{q^{2}}{2} \sum_{j, k} G_{s}\left(\mathbf{r}_{j}, \mathbf{r}_{k}\right) \\
\quad+\sum_{k}\left[u\left(\mathbf{r}_{k}\right)-\frac{q^{2}}{2} G_{s}\left(\mathbf{r}_{k}, \mathbf{r}_{k}\right)\right]+\frac{1}{2} \int d \mathbf{r} d \mathbf{r}^{\prime} \Sigma(\mathbf{r}) G_{s}\left(\mathbf{r}, \mathbf{r}^{\prime}\right) \Sigma\left(\mathbf{r}^{\prime}\right)
\end{aligned}
$$


where $u$ is a one-body interaction energy, which is felt by each counterion due to the presence of the fixed charge and dielectric heterogeneities in the system, and is given by

$$
u(\mathbf{r})=q \int d \mathbf{r}^{\prime} G_{s}\left(\mathbf{r}, \mathbf{r}^{\prime}\right) \Sigma\left(\mathbf{r}^{\prime}\right)+\frac{q^{2}}{2} \Delta G_{0}(\mathbf{r}, \mathbf{r})-\frac{q^{2}}{2} \mathcal{P} G_{\text {free }}(\mathbf{r}, \mathbf{r})+u_{\text {ext }}(\mathbf{r})
$$

The first term is the short-wavelength interaction of the point charge with the fixed charges, while the second term is the interaction of the point charges with the "image charges" generated by the dielectric heterogeneities. The third term is the energy of the long-wavelength correlation hole.

By performing the Hubbard-Stratonovich transformation ${ }^{45,46}$ on both the long-wavelength and short-wavelength electrostatic interactions, the grand partition function of the system can be written as a functional integral over a field $\psi_{s}$ which fluctuates on short lengthscales and a field $\psi_{l}$ which fluctuates on long lengthscales. The integration over $\psi_{s}$ is approximated using a first order cumulant expansion, and the integration over $\psi_{l}$ is evaluated using the mean-field approximation. With these approximations, the Helmholtz free energy $F$ becomes ${ }^{42,43}$

$$
\begin{aligned}
\beta F[\rho, \Sigma] \approx \int & d \mathbf{r} \rho(\mathbf{r})\left[\ln \rho(\mathbf{r}) \Lambda^{d}-1\right]+\int d \mathbf{r} \rho(\mathbf{r}) \beta u(\mathbf{r}) \\
& -\frac{1}{2 \beta} \int d \mathbf{r} d \mathbf{r}^{\prime} i \bar{\psi}_{l}(\mathbf{r}) G_{l}^{-1}\left(\mathbf{r}, \mathbf{r}^{\prime}\right) i \bar{\psi}_{l}\left(\mathbf{r}^{\prime}\right)+\int d \mathbf{r}[q \rho(\mathbf{r})+\Sigma(\mathbf{r})] i \bar{\psi}_{l}\left(\mathbf{r}^{\prime}\right) \\
& +\frac{\beta}{2} \int d \mathbf{r} d \mathbf{r}^{\prime} \Sigma(\mathbf{r}) G_{s}\left(\mathbf{r}, \mathbf{r}^{\prime}\right) \Sigma\left(\mathbf{r}^{\prime}\right)
\end{aligned}
$$

where $\rho(\mathbf{r})$ is the number density of the mobile ions, $\Lambda$ is their thermal wavelength, and $\bar{\psi}_{l}(\mathbf{r})$ is the mean value of the long-wavelength field. The first two terms represent the free energy of the counterions interacting with the one-body energy. The third term is the free energy of the long-wavelength electrostatic field, while the fourth term is the coupling energy between the counterions and the long-wavelength electrostatic field. The final term is the short-range electrostatic interaction energy of the fixed charge distribution in the system.

The mean value of the long-wavelength field $\bar{\psi}_{l}$ is given by

$$
\frac{\delta F}{\delta i \bar{\psi}_{l}(\mathbf{r})}=0
$$

which leads to

$$
-\frac{1}{4 \pi} \nabla \cdot \varepsilon(\mathbf{r}) \nabla \phi(\mathbf{r})=\Sigma(\mathbf{r})+q \rho(\mathbf{r})
$$


where $i \bar{\psi}_{l}=\mathcal{P} \beta \phi$.

In an exact description, the properties of the system are independent of the splitting parameter $\sigma$. However, as the functional integrals entering Eq. (22) are approximate ones, the values of predicted properties of the system do depend on $\sigma$. In order to minimize this dependence, the value of the splitting parameter $\sigma$ is determined through

$$
\frac{\partial F}{\partial \sigma}=0
$$

Consequently, to first order in $\sigma$, the predicted properties are independent of the splitting parameter.

We can now express any equilibrium property of the system from the free energy functional given in Eq. (22). For example, the dimensionless chemical potential $\gamma$ of the counterions is given by

$$
\begin{aligned}
\gamma(\mathbf{r}) & =\frac{\delta}{\delta \rho(\mathbf{r})} \beta F[\rho, \Sigma] \\
& =\ln \rho(\mathbf{r}) \Lambda^{d}+\beta u(\mathbf{r})+q i \bar{\psi}_{l}(\mathbf{r}),
\end{aligned}
$$

and the internal energy of the system by

$$
\begin{aligned}
U & =\frac{\partial}{\partial \beta} \beta F[\rho, \Sigma] \\
& =\int d \mathbf{r} \rho(\mathbf{r}) u(\mathbf{r})+\frac{1}{2} \int d \mathbf{r}[q \rho(\mathbf{r})+\Sigma(\mathbf{r})] i \bar{\psi}_{l}(\mathbf{r})+\frac{1}{2} \int d \mathbf{r} d \mathbf{r}^{\prime} \Sigma(\mathbf{r}) G_{s}\left(\mathbf{r}, \mathbf{r}^{\prime}\right) \Sigma\left(\mathbf{r}^{\prime}\right)
\end{aligned}
$$

\section{B. Application to the spherical cell model}

In this section, we apply the splitting theory described in the Section IV A to the spherical cell model described in Section II B. Thus, the system consists of a central spherical macroion of radius $R_{M}$ with a dielectric constant $\varepsilon^{\prime}$ and its counterions, which are immersed in a medium of dielectric constant $\varepsilon$.

For a fixed and uniform charge distribution on a spherical shell of radius $R_{M}$, the shortwavelength contribution to the self energy of the macroion is

$$
\frac{1}{2} \int d \mathbf{r} d \mathbf{r}^{\prime} \Sigma(\mathbf{r}) G_{s}\left(\mathbf{r}, \mathbf{r}^{\prime}\right) \Sigma\left(\mathbf{r}^{\prime}\right)=\frac{Q^{2}}{2 \sqrt{3} \varepsilon \sigma}\left(\frac{\sigma}{R_{M}}\right)^{2}\left[1-e^{-\sqrt{3} R / \sigma} \cos \left(\frac{R_{M}}{\sigma}\right)\right]
$$


The interior of the spherical macroion has a dielectric constant of $\varepsilon^{\prime}$. The resulting shift of the Green's function, owing to $\varepsilon^{\prime} \neq \varepsilon$, becomes

$$
\Delta G_{0}(\mathbf{r}, \mathbf{r})=\frac{1}{\varepsilon R_{M}} \sum_{l=1}^{\infty}\left(\frac{1-\eta}{1+1 / l+\eta}\right)\left(\frac{R_{M}}{r}\right)^{2 l+2}
$$

where $\eta \equiv \varepsilon^{\prime} / \varepsilon$. Note, in the equation following Eq. (46) in Ref. 10 the factor 2 should be omitted. The one-body interaction energy of the counterions is given by

$$
\begin{aligned}
u(\mathbf{r})= & \frac{q Q}{\sqrt{3} \varepsilon \sigma} \frac{\sigma^{2}}{r R_{M}}\left[e^{-\frac{\sqrt{3}}{2} \frac{r-R_{M}}{\sigma}} \cos \left(\frac{r-R_{M}}{2 \sigma}\right)-e^{-\frac{\sqrt{3}}{2} \frac{r+R_{M}}{\sigma}} \cos \left(\frac{r+R_{M}}{2 \sigma}\right)\right] \\
& +\frac{q^{2}}{2 \varepsilon r} \sum_{l=1}^{\infty} \frac{1-\eta}{1+1 / l+\eta}\left(\frac{R_{M}}{r}\right)^{2 l+1}-\frac{q^{2}}{2 \sqrt{3} \varepsilon \sigma}+u_{\mathrm{ext}}(r) .
\end{aligned}
$$

where the external potential energy $u_{\text {ext }}$ is given in Eq. (14).

For the spherical cell model, the free energy given in Eq. (22) reduces to

$$
\begin{aligned}
\beta F[\rho, \Sigma] \approx \int_{R_{M}}^{R_{\text {cell }}} 4 \pi r^{2} d r \rho(r)\left[\ln \rho(r) \Lambda^{d}-1\right]+\int_{R_{M}}^{R_{\text {cell }}} 4 \pi r^{2} d r \rho(r) \beta u(r) \\
\quad-\frac{\varepsilon}{8 \pi} \int_{R_{M}}^{R_{\text {cell }}} 4 \pi r^{2} d r i \bar{\psi}_{l}^{\prime}(r) \phi^{\prime}(r)+\int_{R_{M}}^{R_{\text {cell }}} 4 \pi r^{2} d r q \rho(r) i \bar{\psi}_{l}(r)+Q i \bar{\psi}_{l}\left(R_{M}\right) \\
\quad+\frac{\beta}{2} \int d \mathbf{r} d \mathbf{r}^{\prime} \Sigma(\mathbf{r}) G_{s}\left(\mathbf{r}, \mathbf{r}^{\prime}\right) \Sigma\left(\mathbf{r}^{\prime}\right)
\end{aligned}
$$

Minimizing the free energy with respect to the long-wavelength field $\bar{\psi}_{l}$ leads to Poisson's equation in spherical coordinates [cf. Eq. (24)]

$$
\varepsilon\left[r^{2} \phi^{\prime}(r)\right]^{\prime}=-4 \pi r^{2} q \rho(r)
$$

with the boundary conditions

$$
\phi^{\prime}\left(R_{M}\right)=-\frac{Q}{\varepsilon R_{M}^{2}} \quad \text { and } \quad \phi^{\prime}\left(R_{\text {cell }}\right)=0
$$

The long-wavelength field is obtained from the electric potential through the relation $\bar{\psi}_{l}=\mathcal{P} \beta \phi$.

The counterion density $\rho(r)$ is related to $i \bar{\psi}_{l}$ through [see Eq. (26)]

$$
\rho(r)=\Lambda^{-d} e^{\gamma-\beta u(r)-q i \bar{\psi}_{l}(r)}
$$


The value of the chemical potential $\gamma$ is determined by ensuring that the total number of counterions in the cell neutralizes the macroion charge.

Within the cell model, the pressure of the system can be obtained from the free energy by the relation

$$
\beta p=-\frac{\partial(\beta F)}{\partial V}=-\frac{1}{4 \pi R_{\text {cell }}^{2}} \frac{\partial(\beta F)}{\partial R_{\text {cell }}} .
$$

Using the expression for the free energy given in Eq. (31), we find that the pressure predicted by the splitting theory is

$$
\beta p=\rho\left(R_{\text {cell }}\right) .
$$

Therefore, we see that the splitting theory, like the PB theory, obeys the contact value theorem, which is exact ${ }^{47}$.

\section{SIMULATION DETAILS}

\section{A. General}

The Monte Carlo (MC) simulations of the four systems as defined in Section III were performed in the canonical ensemble, i.e., at constant number of particles, volume, and temperature. Each simulation involved $10^{7} \mathrm{MC}$ trial moves per counterion. The value of trial displacements were selected differently for the four systems. Counterion distribution functions were determined by using a histogram width of $0.01 R_{M}$ (464 bins). The uncertainties are smaller than the size of the symbols used to present the simulation data in Section VI. However, in the logarithmic plots the first points appear significantly shifted upwards owing to the very rapid density variation at $r \approx R_{M}$. As that does not affect our conclusions, corrections of those shifts have not been made. Statistical uncertainties were calculated using block averaging by subdividing each simulation into twenty equally sized blocks. The integrated $\mathrm{MC} / \mathrm{molecular}$ dynamic/Brownian dynamics simulation package MOLSIM for molecular systems was employed ${ }^{48}$. 


\section{B. Evaluation of the polarization energy}

We have employed a novel, efficient, and accurate scheme to evaluate the polarization energy

$$
U_{\mathrm{pol}}=\frac{1}{2} \sum_{j, k} u_{\mathrm{pol}}\left(\mathbf{r}_{j}, \mathbf{r}_{k}\right)
$$

with $u_{\mathrm{pol}}\left(\mathbf{r}_{j}, \mathbf{r}_{k}\right)$ given by Eq. (15).

First, the polarization energy $U_{\text {pol }}$ can be expressed in several, mathematically equivalent, but numerically different ways. Employing the simplifying notation

$$
\eta \equiv \frac{\varepsilon^{\prime}}{\varepsilon}
$$

and

$$
t_{j k} \equiv \frac{R_{d}}{r_{j}} \frac{R_{d}}{r_{k}}
$$

we obtain:

(A) After introducing the multipole moment $Q_{l m}$ defined by

$$
Q_{l m} \equiv \sum_{k} \frac{q_{k}}{r_{k}^{l+1}} Y_{l m}\left(\theta_{k}, \phi_{k}\right)
$$

we have

$$
U_{\mathrm{pol}}=\frac{1}{2 \varepsilon} \sum_{l=1}^{\infty} \sum_{m=-l}^{l} \frac{4 \pi}{2 l+1}\left[\frac{1-\eta}{1+1 / l+\eta}\right] R_{d}^{2 l+1} Q_{l m} Q_{l m}^{*} .
$$

(B) After denoting the angle between the vectors connecting the positions $\mathbf{r}_{j}$ and $\mathbf{r}_{k}$ by $\gamma_{j k}$ and applying the addition theorem for spherical harmonics, we get

$$
u_{\mathrm{pol}}\left(\mathbf{r}_{j}, \mathbf{r}_{k}\right)=\frac{q_{j} q_{k}}{\varepsilon R_{d}} \sum_{l=1}^{\infty}\left[\frac{1-\eta}{1+1 / l+\eta}\right] t_{j k}^{l+1} P_{l}\left(\cos \gamma_{j k}\right)
$$

where $P_{l}$ is the $l^{\text {th }}$ Legendre polynomial. 
(C) After introducing the exact expansion

$$
\begin{aligned}
\frac{1-\eta}{1+1 / l+\eta}= & \frac{1-\eta}{1+}-\frac{1-\eta}{(1+\eta)^{2}} \frac{1}{l+1} \\
& -\frac{\eta(1-\eta)}{(1+\eta)^{2}} \frac{1}{(1+1 / l+\eta)(1+1 / l)}\left(\frac{1}{l}\right)^{2}
\end{aligned}
$$

and using the generating function of the Legendre polynomial, Eq. (39) can be re-written in a more quickly convergent sum according to

$$
u_{\mathrm{pol}}\left(\mathbf{r}_{j}, \mathbf{r}_{k}\right)=u_{\mathrm{pol}}^{(\mathrm{I})}\left(\mathbf{r}_{j}, \mathbf{r}_{k}\right)+u_{\mathrm{pol}}^{(\mathrm{II})}\left(\mathbf{r}_{j}, \mathbf{r}_{k}\right)+u_{\mathrm{pol}}^{(\mathrm{III})}\left(\mathbf{r}_{j}, \mathbf{r}_{k}\right)
$$

with

$$
\begin{aligned}
u_{\mathrm{pol}}^{(\mathrm{I})}\left(\mathbf{r}_{j}, \mathbf{r}_{k}\right) & =\frac{q_{j} q_{k}}{\varepsilon R_{d}} \frac{1-\eta}{1+\eta} t_{j k}\left(1-2 t_{j k} \cos \gamma_{j k}+t_{j k}^{2}\right)^{-1 / 2} \\
u_{\mathrm{pol}}^{(\mathrm{II})}\left(\mathbf{r}_{j}, \mathbf{r}_{k}\right) & =\frac{q_{j} q_{k}}{\varepsilon R_{d}} \frac{1-\eta}{(1+\eta)^{2}} \ln \frac{1-\cos \gamma_{j k}}{\left[\left(1-2 t_{j k} \cos \gamma_{j k}+t_{j k}^{2}\right)^{1 / 2}+t_{j k}-\cos \gamma_{j k}\right]} \\
u_{\mathrm{pol}}^{(\mathrm{III})}\left(\mathbf{r}_{j}, \mathbf{r}_{k}\right) & =\frac{q_{j} q_{k}}{\varepsilon R_{d}} \frac{1-\eta}{(1+\eta)^{2}} \eta\left\{-t_{j k}-\sum_{l=1}^{\infty} \frac{1}{l^{2}}\left[\frac{1}{1+1 / l+\eta}\right] \frac{t_{j k}^{l+1}}{1+1 / l} P_{l}\left(\cos \gamma_{j k}\right)\right\}
\end{aligned}
$$

For self-terms, $j=k$, we have $\cos \gamma_{k k}=1$, and Eqs. (42)-(44) result to

$$
\begin{aligned}
u_{\mathrm{pol}}^{(\mathrm{I})}\left(\mathbf{r}_{k}, \mathbf{r}_{k}\right) & =\frac{q_{j} q_{k}}{\varepsilon R_{d}} \frac{1-\eta}{1+\eta} \frac{t_{k k}}{1-t_{k k}} \\
u_{\mathrm{pol}}^{(\mathrm{II})}\left(\mathbf{r}_{k}, \mathbf{r}_{k}\right) & =\frac{q_{j} q_{k}}{\varepsilon R_{d}} \frac{1-\eta}{(1+\eta)^{2}} \ln \left(1-t_{k k}\right) \\
u_{\mathrm{pol}}^{(\mathrm{III})}\left(\mathbf{r}_{k}, \mathbf{r}_{k}\right) & =\frac{q_{j} q_{k}}{\varepsilon R_{d}} \frac{1-\eta}{(1+\eta)^{2}} \eta\left\{-t_{k k}-\sum_{l=1}^{\infty} \frac{1}{l^{2}}\left[\frac{1}{1+1 / l+\eta}\right] \frac{t_{k k}^{l+1}}{1+1 / l}\right\}
\end{aligned}
$$

Second, the number of mathematical operations involved in scheme (A) scales as $\mathcal{O}\left(N\left(l_{\max }^{\prime}\right)^{2}\right)$, (B) as $\mathcal{O}\left(N^{2} l_{\text {max }}^{\prime}\right)$, and (C) as $\mathcal{O}\left(N^{2} l_{\text {max }}\right)$ with $N$ being the number of counterions. This shows that, for systems with few ions, scheme (B) is preferred over (A); the opposite holds for a large number of ions. However, the re-expression of the sum over $l$ in scheme (C) makes $l_{\max } \ll l_{\max }^{\prime}$ for the same accuracy, where $l_{\max }^{\prime}$ denotes the the largest $l$ used in Eqs. (38) or (39) and $l_{\max }$ the largest $l$ used in Eq. (44). From the data given in Table II, we found convergence already for $l_{\max }=0$, and hence the scaling of the polarization interaction also becomes $\mathcal{O}\left(N^{2}\right)$, as for the 
Coulombic ion-ion interaction [cf. Eqs. (11) and (13)].

The polarization energy of the heterogeneous dielectric solution was evaluated using scheme (C), i.e., Eqs. (36) and (41)-(44). Table II shows that the error of truncating Eq. (44) at $l_{\max }=0$ is comparable with the statistical uncertainty of the simulations. Our experience is that including the polarization energy using this scheme lengthens the MC simulations roughly by a factor of two.

The expressions given in scheme $(\mathrm{C})$ are formally exact. In special case where $\eta=0$, the infinite series in Eq. (44) does not need to be evaluated, and the polarization energy reduces to the closed form expression previously obtained in Ref. 23, which is exact. The approximate expression for the polarization energy given in Eqs. (5)-(9) in Ref. 23 is related to that in scheme (C), with the exception of: (i) an extra factor of $1+\eta$ in Eq. (43), and (ii) the neglect of the term given by Eq. (44).

TABLE II. Reduced electrostatic interaction energy $U /\left[(N+1) k_{B} T\right]^{a}$ of the four systems examined by the splitting theory and Monte Carlo simulations using different $l_{\max }$.

\begin{tabular}{ccccccc}
\hline \hline \multicolumn{2}{l}{ Method Dielec. ${ }^{b} l_{\max }{ }^{c}$} & \multicolumn{4}{c}{ System } \\
& & & I & II & III & IV \\
\hline Theory & Homo & - & -0.901 & -13.9 & -31.2 & -0.847 \\
& Hetero & - & -0.857 & -13.0 & -28.1 & -0.835 \\
\hline MC & Homo & - & $-0.8016(8)$ & $-13.2579(7)$ & $-30.260(1)$ & $-0.8089(2)$ \\
& Hetero & 0 & $-0.7496(6)$ & $-12.2856(6)$ & $-27.010(1)$ & $-0.7929(2)$ \\
& Hetero & 1 & $-0.7485(5)$ & $-12.2854(5)$ & $-27.010(1)$ & $-0.7931(2)$ \\
& Hetero & 2 & $-0.7484(6)$ & $-12.2858(6)$ & $-27.009(1)$ & $-0.7935(2)$ \\
\hline \hline
\end{tabular}

${ }^{a}$ Statistical uncertainty (one standard deviation) in parenthesis.

${ }^{b}$ Homo: $\varepsilon^{\prime}=78.4026$ and Hetero: $\varepsilon^{\prime}=1$.

${ }^{c}$ Upper limit of the sum in Eq. (44).

\section{RESULTS}

Properties of the four colloidal systems obtained by applying the splitting theory and by Monte Carlo simulations will be presented together with some complementary results from the PB equation. Results of homogeneous dielectric systems will be given first and followed by results of heterogeneous dielectric systems. 


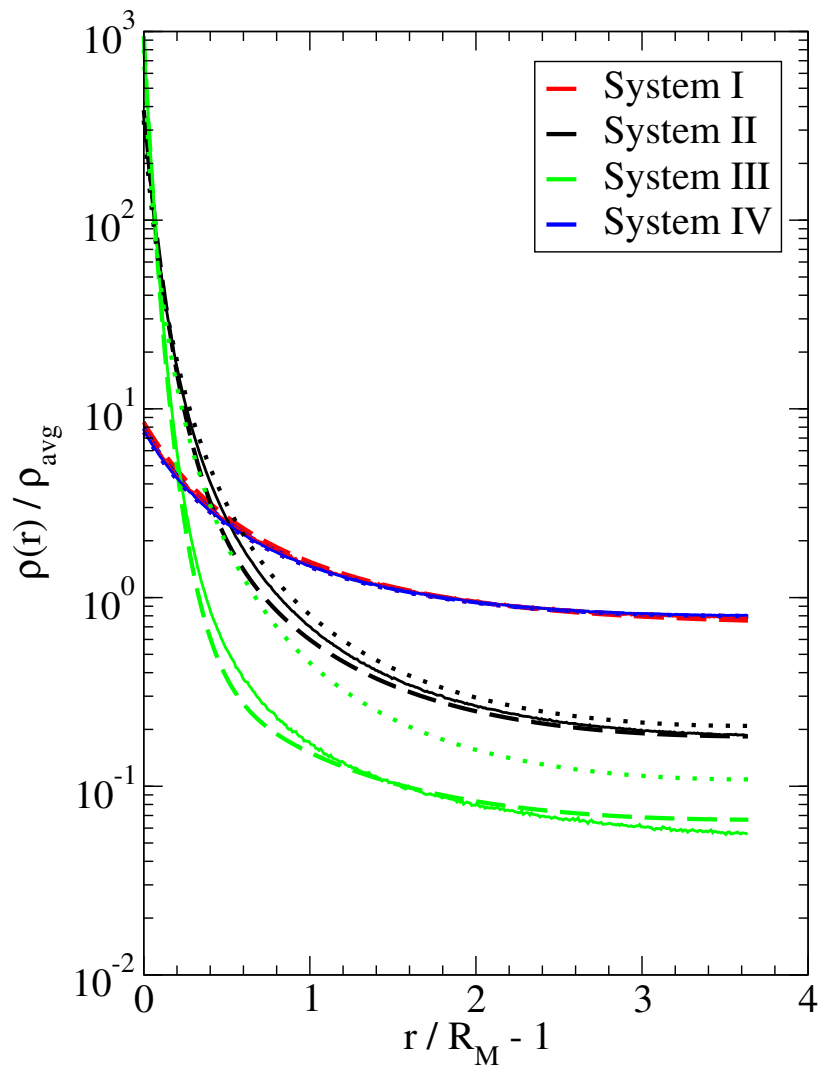

FIG. 1. Normalized counterion number density $\rho(r) / \rho_{\text {avg }}$ as a function of the scaled radial distance $r / R_{M}$ from the center of the macroion for System I (red), System II (black), System III (green), and System IV (blue) as obtained from the Poisson-Boltzmann theory (data from Ref. 44) (dotted curves), the splitting theory (dashed curves), and Monte Carlo simulations (solid curves). The results of Systems I and IV of the PB equation are identical and all data for these two systems essentially collapse on a single curve.

\section{A. Homogeneous dielectric solution}

We will here examine the case where the dielectric constant of the macroions $\varepsilon^{\prime}$ is the same as that of the surrounding solvent $\varepsilon$, i.e., $\varepsilon^{\prime}=\varepsilon$. Figure 1 shows the normalized counterion number density as a function of the radial position for the four systems described in Section III and investigated using the PB theory ${ }^{44}$ (dotted curves), the splitting theory described in Section IV (dashed curves), and Monte Carlo simulations described in Section V (solid curves). The corresponding values of the splitting parameter $\sigma$ are given in Table I for these homogeneous dielectric systems. Table II provides the electrostatic interaction energy of the four systems.

Generally, there is an accumulation of the counterions near the macroion with the maximal density appearing at contact $r=R_{M}$ (Fig. 1), arising from the attractive electrostatic macroioncounterion interaction. In Systems I and IV, the counterion distributions deviate only moderately 
from a uniform distribution, which originates from the fact that the electrostatic coupling parameter $\Xi$ is comparably small. The distribution of the counterions becomes much more nonuniform in Systems II and III, where $\Xi=5$ and 40 , respectively.

The predictions of the PB theory agree well with the MC simulation data for Systems I and IV, possessing low values of $\Xi$. At larger values of $\Xi$, the PB approximation underestimates the accumulation of the small counterions near the macroion, leading to a density that is too large further away. Noticeably, within the spherical cell model, the osmotic pressure is given by $\beta p=\rho\left(R_{\text {cell }}\right)$, so the PB approximation overestimates the osmotic pressure at large $\Xi$.

The length $\sigma$, entering in the splitting operator $\mathcal{P}$ of the splitting theory, can be interpreted as the size of a correlation hole around each counterion. The optimal $\sigma$ as a function of the electrostatic coupling parameter $\Xi$ is given in Fig. 2 for three different colloidal sizes $R_{M}$. In the strong-coupling limit $(\Xi \gg 1)$, the dependence of the free energy of the system on the splitting parameter is dominated by the one-body energy of the counterions [second term in Eq. (22)] and the short-range electrostatic interaction energy of the macroion charge [last term in Eq. (22)]. The balance between these two terms leads ${ }^{42,43}$ to the scaling relation $\sigma \propto \Xi^{-1 / 2}$ with a prefactor basically independent of $R_{M} / l_{B}$ (Fig. 2).

The splitting parameter diverges at $\Xi^{*}=|q| l_{B}^{2} /\left(2 R_{M}^{2}\right)$, which is the value of the coupling parameter which corresponds to a macroion with $Q=-q$. In the limit $\sigma \rightarrow \infty, G_{l}=0$ and $G_{s}=G_{0}$, and thus the splitting theory reduces to an exact description of a macroion with a single counterion ion, where the density profile becomes $\rho(r) \propto e^{-\beta q Q /(\varepsilon r)}$. In contrast, the PB equation does not reduce properly to this limit, as it does not account for the discreteness of the counterions. For this particular geometry, the PB theory does not become exact in the weak coupling limit, due to the finite and limited number of counterions in the system. In the weak coupling limit, the PB approximation becomes a good approximation only when the number of counterions remains substantial, i.e., $|Q / q| \gg 1$.

Figure 3 displays the counterion number density profile at different macroion charge $Q$, keeping the macroion radius $R_{M}$, macroion volume fraction $\phi$, and the counterion charge $q$ fixed. This is equivalent to increasing the coupling parameter $\Xi$ at constant $R_{\text {cell }} / R_{M}$ and $R_{M} / l_{B}$. In panel (a), $Q=20,40$, and 100 at $R_{M} / l_{B}=0.702839$, and in panel (b), $Q=80,10^{3}, 10^{4}$, and $10^{5}$ at $R_{M} / l_{B}=22.4909$. As the charge of the macroion is increased, the counterions become increasingly attracted to the macroion surface. In the strong-coupling regime $(\Xi \gg 1)$, we see that there are two distinct regions in the counterion distribution: a tightly bound inner layer, and 


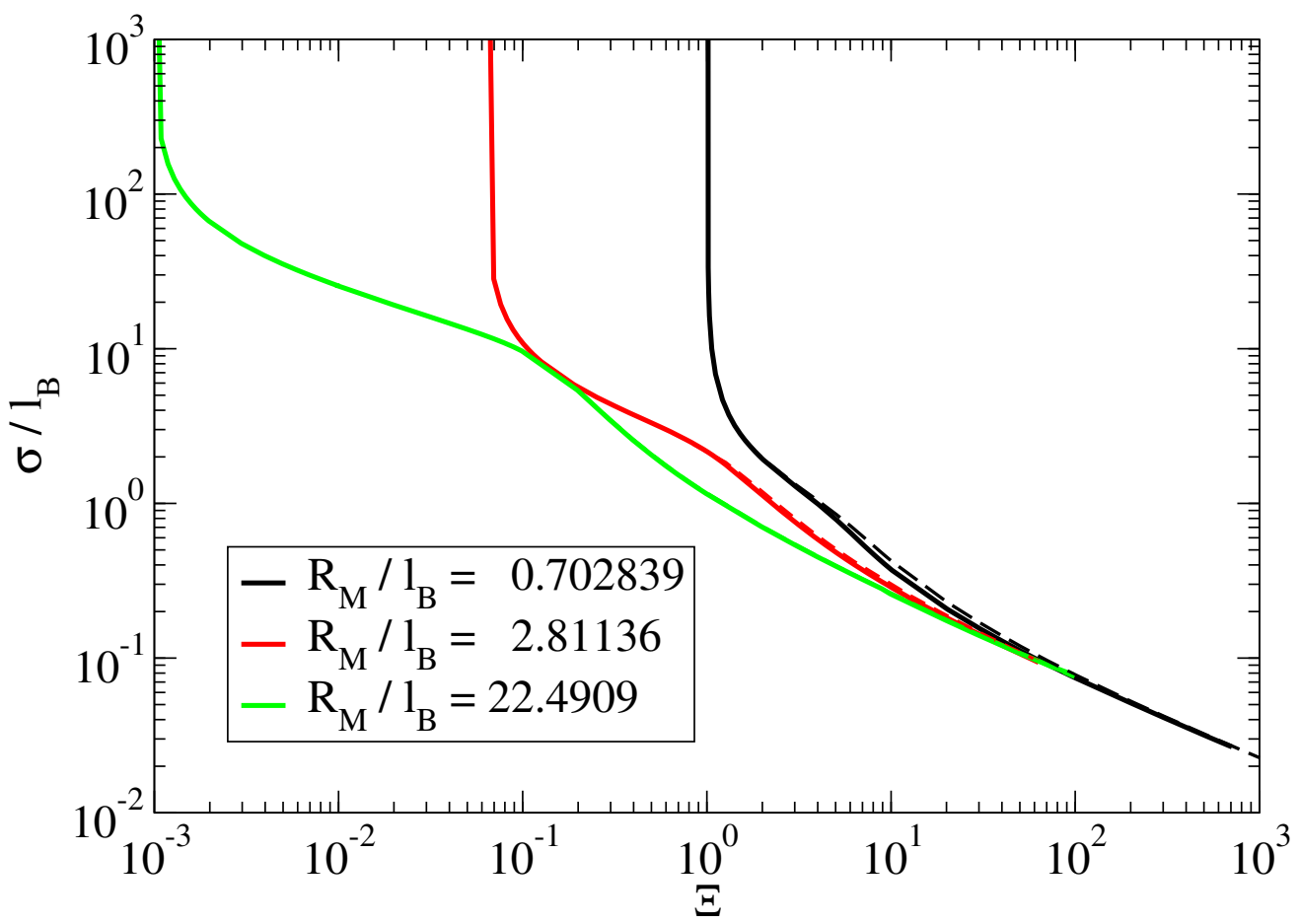

FIG. 2. Reduced splitting parameter $\sigma / l_{B}$ as a function of the electrostatic coupling parameter $\Xi$ for $\eta=1$ (solid curves) and $\eta=1 / 78.4026$ (dashed curves) at $R_{\text {cell }} / R_{M}=4.64$ with $R_{M} / l_{B}=0.702839$ (black curves), $R_{M} / l_{B}=2.81136$ (red curves), and $R_{M} / l_{B}=22.4909$ (green curves). The solid and dashed curves are mostly superimposed.

a diffuse outer layer. The width of the inner layer is of the order of $\sigma$. At the same value of the coupling constant, the counterions are more closely associated to macroions of larger size (cf. Figs. $3 \mathrm{~b}$ and $3 \mathrm{a}$ ). This is due to the smaller repulsions between the counterions bound to the larger macroion because they are, on average, further apart.

\section{B. Heterogeneous dielectric solution}

Now, we assess the ability of the splitting theory to describe counterion distributions when the macroion possesses a dielectric constant $\varepsilon^{\prime}=1$, while keeping the dielectric constant of the solvent at $\varepsilon=78.4026$. The radial distribution of the counterions around the macroion for each of the four systems examined are given in Fig. 4, as predicted by the splitting theory (lines) and by Monte Carlo simulation (symbols), presented using a linear (left column) and logarithmic (right column) abscissa. The corresponding data for $\varepsilon^{\prime}=78.4026$ are also included.

Generally, while the maxima of the counterion distributions appear at $r=R_{M}$ for a homoge- 

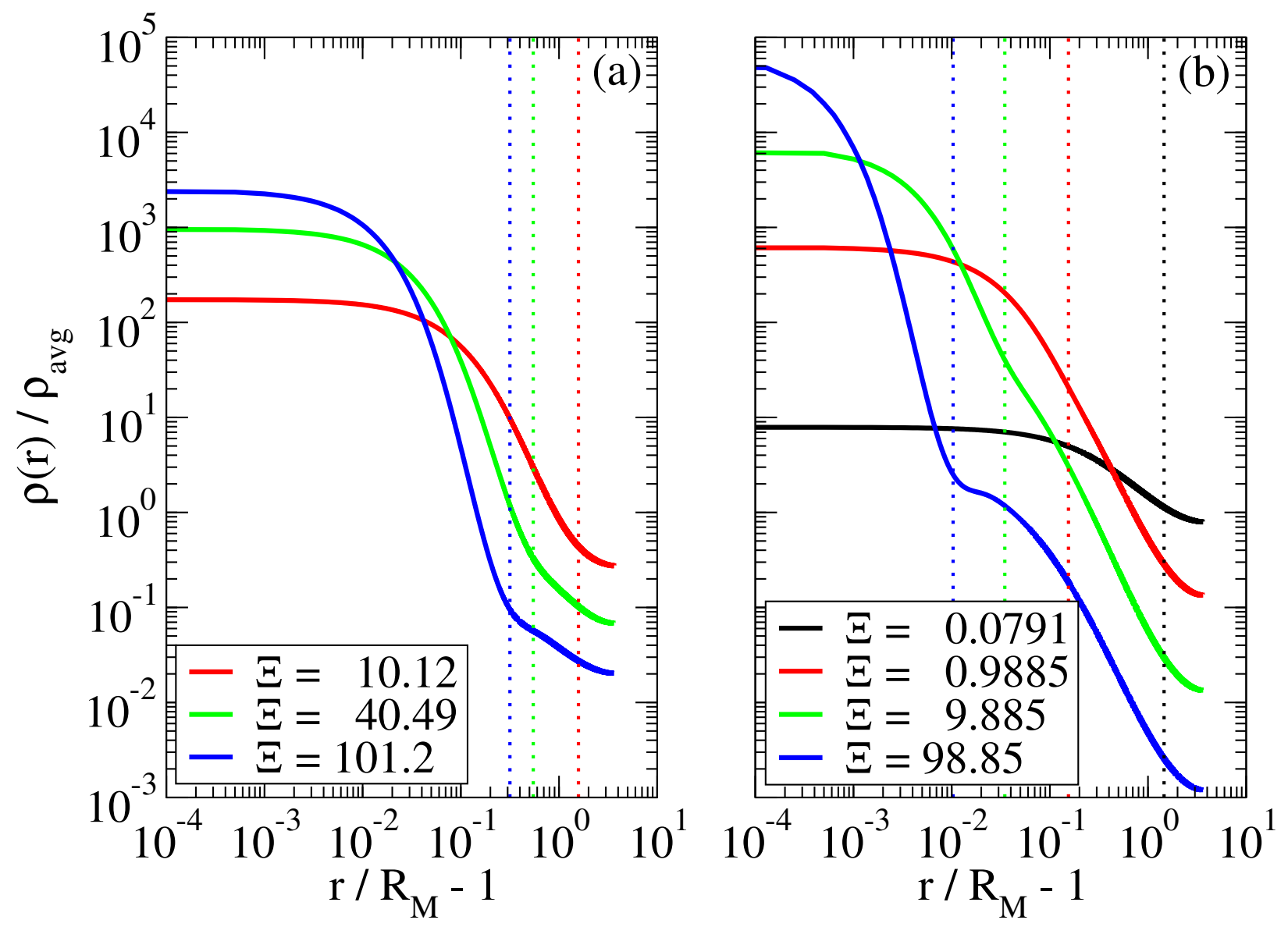

FIG. 3. Normalized counterion number density $\rho(r) / \rho_{\text {avg }}$ as a function of the scaled radial distance $r / R_{M}-$ 1 from the center of the macroion for the radii ratio $R_{\text {cell }} / R_{M}=4.64$ and macroion radius (a) $R_{M} / l_{B}=$ 0.702839 and (b) $R_{M} / l_{B}=22.4909$ for homogeneous $\left(\varepsilon^{\prime}=\varepsilon\right)$ dielectric solutions. In $(\mathrm{a}),(|Q / q|, \Xi)=$ $(10,10.12)$ (red curve), (40, 40.4873) corresponding to System III (green curve), and (100, 101.2) (blue curve); and in (b), $(|Q / q|, \Xi)=(80,0.0791)$ corresponding to System IV (black curve), $\left(10^{3}, 0.9885\right)$ (red curve), $\left(10^{4}, 9.885\right)$ (green curve), and $\left(10^{5}, 98.85\right)$ (blue curve). The locations where $r-R_{M}=3 \sigma$ are also indicated (vertical dotted lines).

neous dielectric permeability, for $\varepsilon^{\prime}<\varepsilon$ (i) the counterion density is zero at $r=R_{M}$ and (ii) the maxima appears $r>R_{M}$. Observation (i) originates from the fact that the additional repulsive potential $U_{\text {pol }}$ operating between the counterions and the induced polarization charges diverges at $r=R_{d}=R_{M}$ [see Eqs. (41)-(44)], and finding (ii) results from the combination of $U_{\text {pol }}$ with the attractive Coulomb macroion-counterion interaction. These principal features agree with those found and discussed in previous publications on the same topic ${ }^{21-23}$.

In more detail, the predictions of the splitting theory agree to a very high degree with the simulation data for Systems I and IV. Only at distances shorter than $0.01 R_{M}$, detectable differences are found (see right column of Fig. 4). In System II, noticeable differences are seen up to $r \approx 1.5 R_{M}$ 

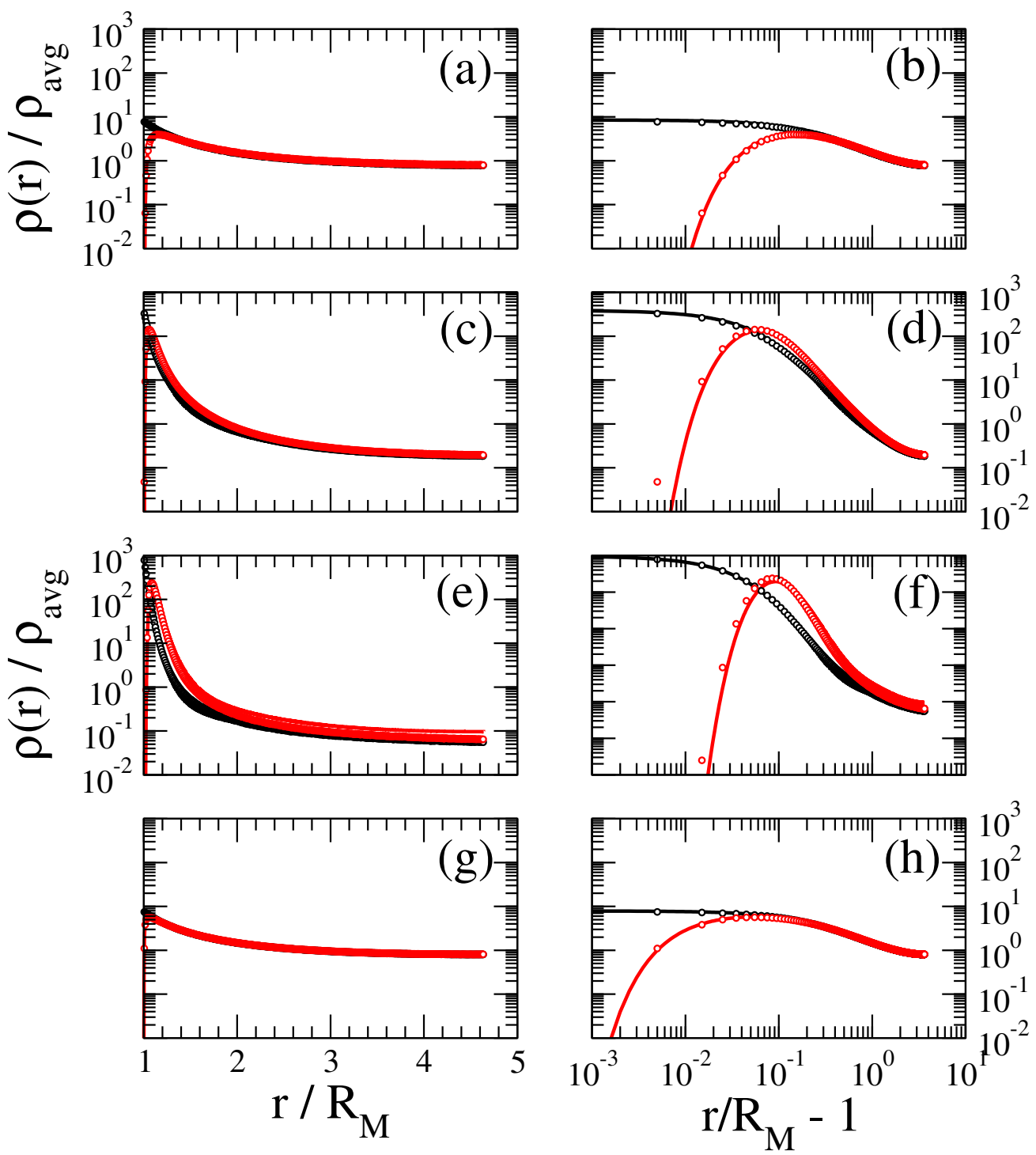

FIG. 4. Normalized counterion number density $\rho(r) / \rho_{\text {avg }}$ as a function of the scaled radial distance $r / R_{M}$ from the center of the macroion for System I (a,b), II (c,d), III (e,f), and IV (g,h) with $\varepsilon^{\prime}=\varepsilon$ (black) and $\varepsilon^{\prime}=1$ (red) as obtained from the splitting theory (curves) and Monte Carlo simulations (symbols). The abscissa is on a linear scale in the left column and on a logarithmic scale in the right column. Results of the splitting theory and Monte Carlo simulations are nearly indistinguishable in the left column.

(Figs. 4c and d). In System III, which has the strongest electrostatic coupling, the prediction of the splitting theory is only qualitatively correct (Fig. $4 \mathrm{e}$ and f). It accurately predicts the location of the peak of the counterion distribution; however, it underestimates the counterion densities at short distances, and overestimates the density at large distances.

Similar systems have been examined recently in Ref. 49, where they develop a modified Poisson-Boltzmann equation to account for the presence of the dielectric interface. This works 
well in the far-field region but not near the interface. When this modified PB equation is coupled together with a theory for the strongly-correlated region near the interface, it accurately describes the counterion density profiles simulated in the work. Interestingly, the splitting theory reduces to their modified PB equation in the limit that $\sigma \rightarrow 0$. This suggests that using a spatially varying splitting parameter could improve the theory developed in this work, particularly the predictions far from the macroion.

Figure 5 displays the radial counterion density profile with macroion charges as for the systems given in Fig. 3. We see that the location of the density maxima (on a relative scale) moves closer to the macroion surface (i) as the macroion charge increases and (ii) as the macroion size increases. The former is due to the increased counterion-macroion Coulomb attraction, and the latter to the decreased polarization repulsion. In the strong-coupling regime, there are also here two distinct layers of counterions.

Finally, the variation of the value of the splitting parameter $\sigma$ with the strength of the electrostatic coupling $\Xi$ was shown in Fig. 2. The values of $\sigma$ for the systems with a dielectric heterogeneity becomes slightly larger, but still close to those for homogeneous dielectric system. The differences in $\sigma$ increase (i) as the Bjerrum length becomes larger than the macroion radius and (ii) as the image charge interactions becomes stronger.

\section{CONCLUSIONS}

We have studied models of charged spherical colloids in salt-free solution, including cases where the dielectric constant of the macroion interior is lower than that of the surrounding solution. A new method for evaluating the polarization energy arising from the dielectric heterogeneity has been developed and employed in Monte Carlo simulations of these systems. This method has been found to converge extremely rapidly and offers great computational advantages over the previous methods (with the exception of the just published work by dos Santos et al. ${ }^{23}$, which shares features with the method presented here), when the number of counterions in the system is sufficiently low.

We have also assessed the accuracy of a recently developed field theory. This splitting theory predicts counterion density profiles that compare well those from Monte Carlo simulations. That holds even in the presence of a dielectric interface; however, the theory overestimates the pressure and potential energy of the system. At high electrostatic couplings, the theory predicts counterion density profiles that qualitatively divide into a two distinct regions: one strongly bound inner layer 

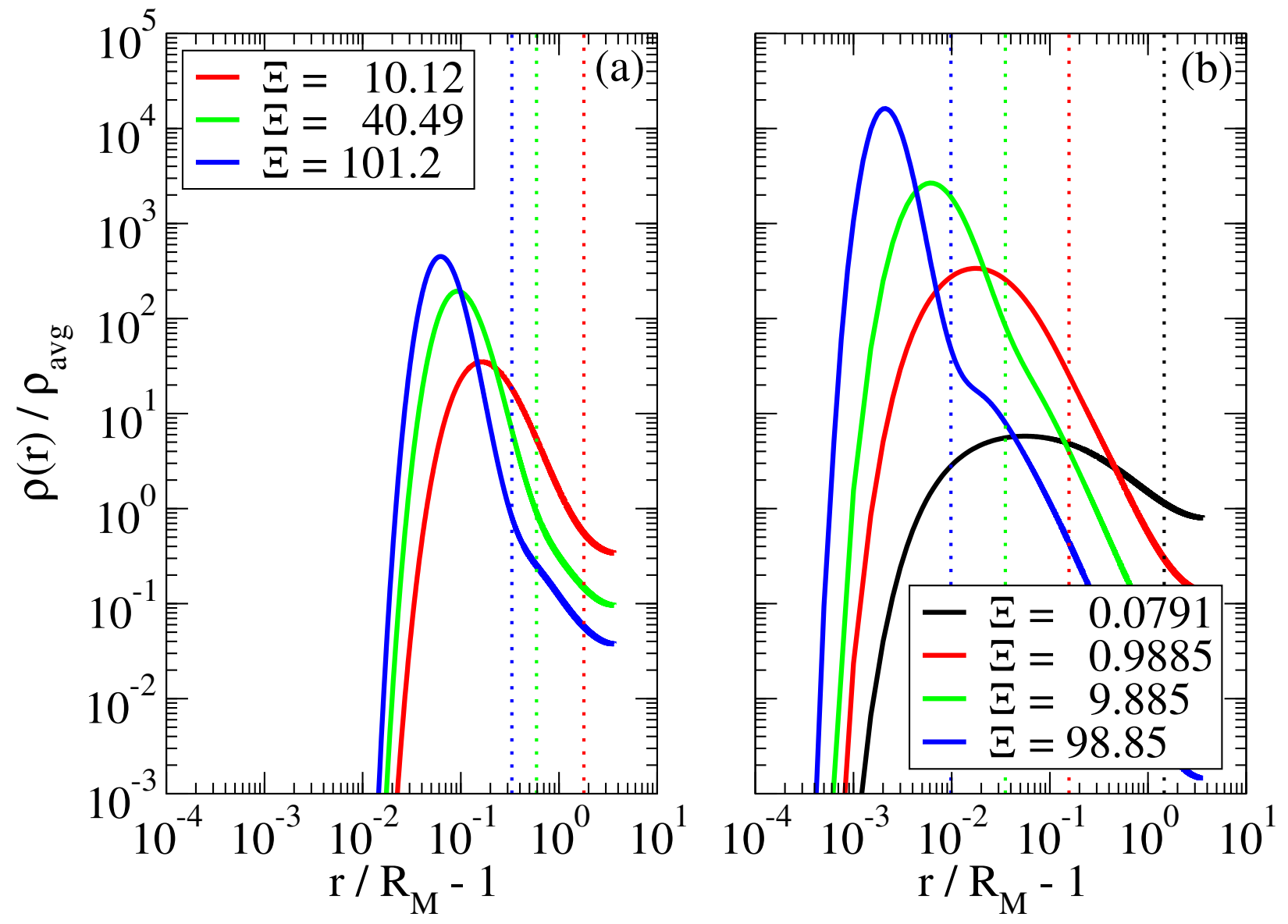

FIG. 5. As in Fig. 3 but for a heterogeneous $\left(\varepsilon^{\prime}=1 \neq \varepsilon\right)$ dielectric solution.

and one diffuse outer layer. The thickness of the bound layer scales with the splitting parameter $\sigma$.

\section{ACKNOWLEDGMENTS}

Financial support by the Swedish Research Council (VR) through the Linnaeus grant Organizing Molecular Matter (OMM) center of excellence (239-2009-6794) and through individual grants to PL (621-2007-5251) are gratefully acknowledged.

\section{REFERENCES}

${ }^{1}$ D. F. Evans and H. Wennerström, The Colloidal Domain: Where Physics, Chemistry, Biology, and Technology Meet, 2nd ed. (Wiley-VCH, New York, 1999).

${ }^{2}$ C. W. Outhwaite and L. B. Bhuiyan, J. Chem. Soc., Faraday Trans. 2 79, 707 (1983). 
${ }^{3}$ C. Outhwaite and L. Bhuiyan, Mol. Phys. 74, 367 (1991).

${ }^{4}$ L. B. Bhuiyan and C. W. Outhwaite, Condens. Matter Phys. 8, 287 (2005).

${ }^{5}$ M. Fushiki, Chem. Phys. Lett. 154, 77 (1989).

${ }^{6}$ E. Gonzalez-Tovar and M. Lozada-Cassou, J. Phys. Chem. 93, 3761 (1989).

${ }^{7}$ G. I. Guerrero-García, E. González-Tovar, M. Lozada-Cassou, and F. de J. Guevara-Rodríguez, J. Chem. Phys. 123, 034703 (2005).

${ }^{8}$ R. D. Coalson and A. Duncan, J. Chem. Phys. 97, 5653 (1992).

${ }^{9}$ R. R. Netz and H. Orland, Eur. Phys. J. E 1, 203 (2000).

${ }^{10}$ R. A. Curtis and L. Lue, J. Chem. Phys. 123, 174702 (2005).

${ }^{11}$ Y.-X. Yu, J. Wu, and G.-H. Gao, J. Chem. Phys. 120, 7223 (2004).

${ }^{12}$ T. Goel and C. N. Patra, J. Chem. Phys. 127, 034502 (2007).

${ }^{13}$ B. I. Shklovskii, Phys. Rev. E 60, 5802 (1999).

${ }^{14}$ A. G. Moreira and R. R. Netz, Phys. Rev. Lett. 87, 078301 (2001).

${ }^{15}$ J. Dobnikar, R. Castañeda-Priego, H. H. von Grünberg, and E. Trizac, New J. Phys. 8, 277 (2006).

${ }^{16}$ E. Trizac and Y. Levin, Phys. Rev. E 69, 031403 (2004).

${ }^{17}$ S. Pianegonda, E. Trizac, and Y. Levin, J. Chem. Phys. 126, 014702 (2007).

${ }^{18}$ T. E. Colla, Y. Levin, and E. Trizac, J. Chem. Phys. 131, 074115 (2009).

${ }^{19}$ T. E. Colla and Y. Levin, J. Chem. Phys. 133 (2010), 10.1063/1.3523349.

${ }^{20}$ M. M. Hatlo and L. Lue, Soft Matter 4, 1582 (2008).

${ }^{21}$ P. Linse, J. Phys. Chem. 90, 6821 (1986).

${ }^{22}$ R. Messina, J. Chem. Phys. 117, 11062 (2002).

${ }^{23}$ A. P. dos Santos, A. Bakhshandeh, and Y. Levin, J. Chem. Phys. 135, 044124 (2011).

${ }^{24}$ J. Reščič and P. Linse, J. Chem. Phys. 129, 114505 (2008).

${ }^{25}$ M. Kanduc, A. Naji, and R. Podgornik, J. Chem. Phys. 132, 224703 (2010).

${ }^{26}$ C. Wagner, Physik. Z. 25, 474 (1924).

${ }^{27}$ L. Onsager and N. N. T. Samaras, J. Chem. Phys. 2, 528 (1934).

${ }^{28}$ J. Groenewold, J. Chem. Phys. 107, 9668 (1997).

${ }^{29}$ R. Kjellander and S. Marcelja, J. Chem. Phys. 82, 2122 (1985).

${ }^{30}$ R. Kjellander and S. Marcelja, Chem. Phys. Lett. 142, 485 (1987).

${ }^{31}$ P. Attard, D. J. Mitchell, and B. W. Ninham, J. Chem. Phys. 88, 4987 (1988).

${ }^{32}$ P. Attard, D. J. Mitchell, and B. W. Ninham, J. Chem. Phys. 89, 4358 (1988). 
${ }^{33}$ R. Netz, Eur. Phys. J. E 5, 189 (2001).

${ }^{34}$ R. Netz, Eur. Phys. J. E 5, 557 (2001).

${ }^{35}$ D. S. Dean and R. R. Horgan, Phys. Rev. E 69, 061603 (2004).

${ }^{36}$ M. M. Hatlo, R. A. Curtis, and L. Lue, J. Chem. Phys. 128, 164717 (2008).

${ }^{37}$ S. Buyukdagli, M. Manghi, and J. Palmeri, J. Chem. Phys. 134, 074706 (2011).

${ }^{38}$ N. Ben-Tal and R. D. Coalson, J. Chem. Phys. 101, 5148 (1994).

${ }^{39}$ Y. S. Jho, M. Kanduc, A. Naji, R. Podgornik, M. W. Kim, and P. A. Pincus, Phys. Rev. Lett. 101 (2008), 10.1103/PhysRevLett.101.188101.

${ }^{40}$ A. Arnold and C. Holm, Eur. Phys. J. E 27, 21 (2008).

${ }^{41}$ A. Naji and R. R. Netz, Europhys. J. E 13, 43 (2004), 10.1140/epje/e2004-00039-X.

${ }^{42}$ M. M. Hatlo and L. Lue, Soft Matter 5, 125 (2009).

${ }^{43}$ M. M. Hatlo and L. Lue, EPL (Europhysics Letters) 89, 25002 (2010).

${ }^{44} \mathrm{P}$. Linse, in Advanced Computer Simulation Approaches for Soft Matter Sciences II, Advances in Polymer Science, Vol. 185, edited by C. Holm and K. Kremer (Springer Berlin / Heidelberg, 2005) pp. 94-94.

${ }^{45}$ R. L. Stratonovich, Dokl. Akad. Nauk SSSR 115, 1097 (1957).

${ }^{46}$ J. Hubbard, Phys. Rev. Lett. 3, 77 (1959).

${ }^{47}$ H. Wennerström, B. Jönsson, and P. Linse, J. Chem. Phys. 76, 4665 (1982).

${ }^{48}$ P. Linse, MOLSIM, Version 5.0, Lund University, Sweden (2011).

${ }^{49}$ A. Bakhshandeh, A. P. dos Santos, and Y. Levin, Phys. Rev. Lett. 107, 107801 (2011). 